\title{
A new deterministic model for chaotic reversals
}

\author{
Christophe Gissinger ${ }^{1}$ \\ 1 Department of Astrophysical Sciences/Princeton Plasma Physics Lab, 5 Peyton Hall, Princeton University, Princeton NJ \\ USA. 19104-2688
}

Received: date / Revised version: date

\begin{abstract}
We present a new chaotic system of three coupled ordinary differential equations, limited to quadratic nonlinear terms. A wide variety of dynamical regimes are reported. For some parameters, chaotic reversals of the amplitudes are produced by crisis-induced intermittency, following a mechanism different from what is generally observed in similar deterministic models. Despite its simplicity, this system therefore generates a rich dynamics, able to model more complex physical systems. In particular, a comparison with reversals of the magnetic field of the Earth shows a surprisingly good agreement, and highlights the relevance of deterministic chaos to describe geomagnetic field dynamics.
\end{abstract}

PACS. XX.XX.XX No PACS code given

\section{Introduction}

Deterministic chaotic models have been largely studied in the past few decades, both on the numerical and theoretical sides [1, 2], 3]. In addition to their interest to nonlinear mathematics, these models often play an important role in the comprehension of physical systems. Indeed, in many situations, they offer a simple modelisation of experimental and natural objects exhibiting complex dynamics. Particular interest has been devoted to models involving only three equations, the minimum number required to generate chaos in a continuous dynamical system with time independent coefficients. Similarly, ordinary differential equations of these types of models are generally restricted to the lowest order terms. This approach ensures that numerical integration yields chaotic dynamics relevant to describe the behaviors of complex systems, meanwhile a theoretical study of the model is still possible.

In this article, we focus on the dynamics of reversals, i.e. the periodic or chaotic changes of sign of the amplitudes in dynamical systems. Reversals between two symmetric states are observed in several natural or experimental systems, among which we can mention thermal convection, magnetohydrodynamics dynamos, or turbulent atmospheric flows. Although these systems generally involve turbulent states and a very large number of degrees of freedom, several sets of deterministic equations have been proposed to model reversals, the Lorenz model providing the most famous example [4. As typical examples of chaotic three-dimensional systems exhibiting reversals, we can also mention the Rossler attractor [5, the Rikitake equations [6] and the Sprott models [7. Such deterministic three-mode equations often provide relevant toy models, but generally fail to describe the details of the dynamics of the modeled system. In the case of reversals occurring in fluids mechanics, in which turbulent fluctuations are important, explanations in terms of stochastic processes are thus usually preferred to nonlinear chaotic dynamics. Here, we propose a new model for reversals, given by the following system of three coupled ordinary differential equations:

$$
\begin{aligned}
& \dot{Q}=\mu Q-V D \\
& \dot{D}=-\nu D+V Q \\
& \dot{V}=\Gamma-V+Q D
\end{aligned}
$$

We will see that the type of dynamics generated by these equations is relatively different from previous nonlinear chaotic models, and is sometimes more similar to stochastic systems. It has been recently proposed as a model for reversals of a turbulent magnetic dynamo obtained in numerical simulations [8]. In the last section of this article, we will discuss a physical interpretation of this model in the framework of reversals of the Earth magnetic field.

In this system of equations, the nonlinear terms are restricted to quadratic order, and the parameters $\mu, \nu$ and $\Gamma$ are real. Relative signs of the quadratic terms are such that the solution remains bounded. The divergence of this vector field is equal to $\mu-\nu-1$, so it is dissipative for $\mu>1+\nu$. This very simple dynamical system presents similarities with several three-modes models previously discussed in the literature. In the context of geodynamo reversals, Rikitake [6] proposed a model close to (1]-3), except that the linear damping of the mode $V$ was discarded. Moreover, in Rikitake model, $D$ and $Q$ were linearly stable. Nozières 9 derived a similar model, also related to geomagnetic reversals, but obtained from a truncation of MHD equations, the physical interpretation of this model being a 

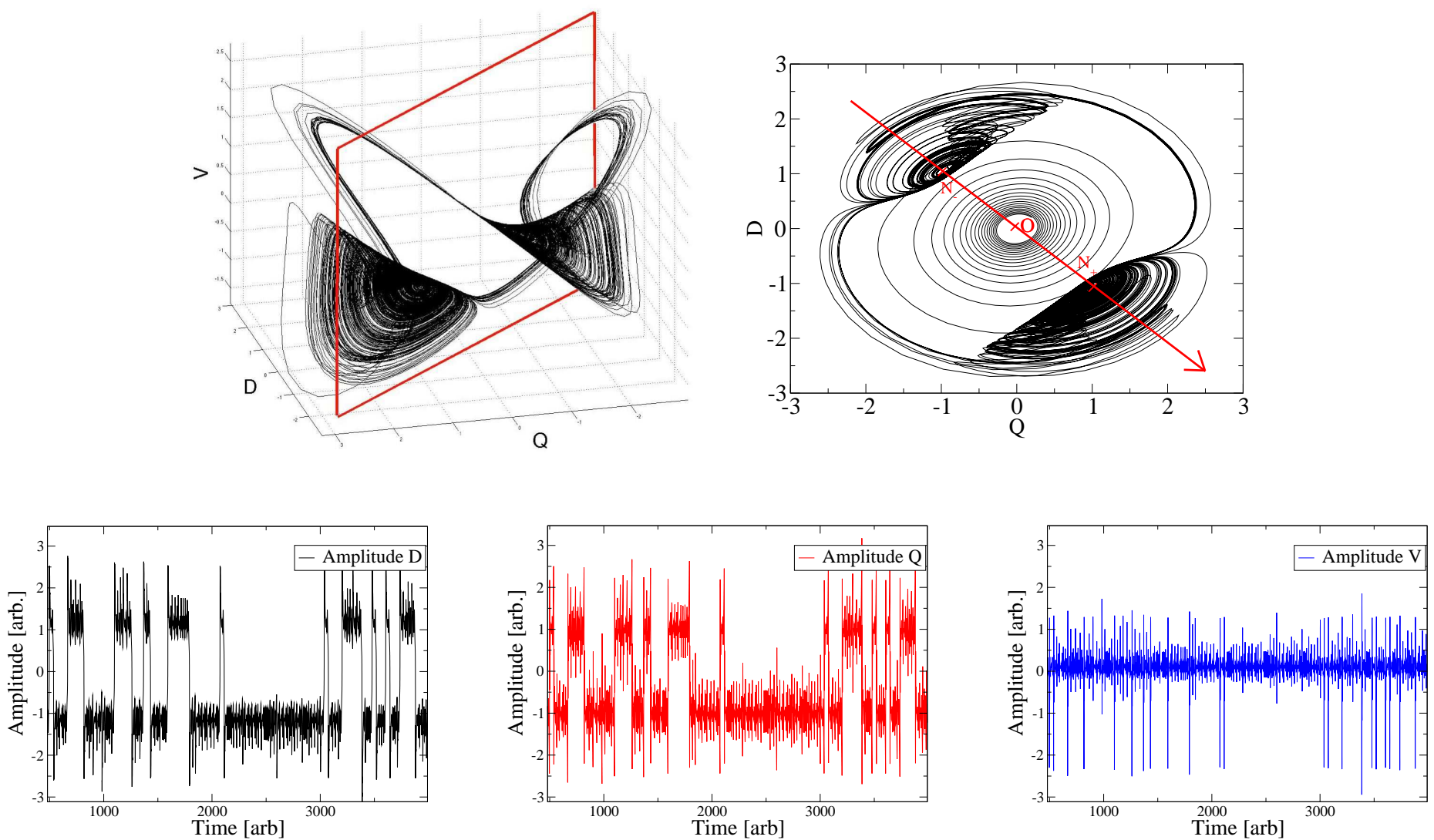

Fig. 1: Numerical integration of the model for $\mu=0.119, \nu=0.1$ and $\Gamma=0.9$. Poincare section used for iterated map is shown in red. Top panel: trajectories in the phase space $(D, Q, V)$ (left) and in the cut $(D, Q)$ (right). Bottom panel: Time evolution of amplitudes $D$ (left), $Q$ (middle) and $V$ (right).

system of two magnetic components coupled to one velocity mode. Therefore, in Nozières model, linear terms represent ohmic decays and the modes are linearly damped, strongly modifying the dynamics. Finally, the dynamical system (1-3) with $\Gamma=0$ is relevant for several hydrodynamical problems, and has been extensively studied in a series of articles by Proctor and Hughes [10]. Again, we will see that a non-zero $\Gamma$ is crucial for the dynamics described in the present paper. Finally, note that the system possesses the symmetry $(D, Q, V) \rightarrow(-D,-Q, V)$, which is crucial for the dynamics of reversals reported in section 3.

\section{Stability and fixed points}

In this section, we first describe the fixed points of the system. A first trivial fixed point $O$ is related to vanishing amplitudes of $D$ and $Q$ :

$$
Q_{O}=0, \quad D_{O}=0, \quad V_{O}=\Gamma
$$

Linearization around this fixed point give the following eigenvalues:

$$
\begin{aligned}
& \lambda_{i}=\frac{\mu-\nu}{2} \pm \frac{1}{2} \sqrt{(\mu+\nu)^{2}-4 \Gamma^{2}}, \quad i=1,2 \\
& \lambda_{3}=-1
\end{aligned}
$$

The trivial eigenvalue $\lambda_{3}$ indicates a stable direction, independently of the values of $\mu, \nu$ and $\Gamma$, and traces back to the linear damping of the amplitude $V$. When $\mu+\nu>2 \Gamma$, all eigenvalues are real. When $\mu+\nu<2 \Gamma$, eigenvalues $\lambda_{1}$ and $\lambda_{2}$ become complex conjugates and the system undergoes Hopf bifurcation for $\mu>\nu$. Note that this system exhibits a co-dimension two bifurcation point for $\mu=\nu=\Gamma$, i.e. the system is close to both a stationary and a Hopf bifurcation. We will see that the behavior of our dynamical system strongly depends on the stability of $O$.

Apart from this trivial point, there are four non-trivial fixed points $M_{\mp}$ and $N_{\mp}$. Two of these solutions only exist when $\sqrt{\mu \nu}-\Gamma>0$ :

$$
\begin{aligned}
& Q_{M \pm}= \pm \sqrt{\nu-\Gamma \sqrt{\frac{\nu}{\mu}}} \\
& D_{M \pm}= \pm \sqrt{\mu-\Gamma \sqrt{\frac{\mu}{\nu}}}
\end{aligned}
$$




$$
V_{M \pm}=\sqrt{\mu \nu}
$$

The other fixed points are :

$$
\begin{gathered}
Q_{N \pm}= \pm \sqrt{\nu+\Gamma \sqrt{\frac{\nu}{\mu}}} \\
D_{N \pm}=- \pm \sqrt{\mu+\Gamma \sqrt{\frac{\mu}{\nu}}} \\
V_{N \pm}=-\sqrt{\mu \nu}
\end{gathered}
$$

Linearization of the system around the fixed points $M_{ \pm}$gives the following characteristic relation:

$\lambda^{3}+\lambda^{2}(1+\nu-\mu)+\lambda\left(\Gamma \frac{(\nu-\mu)}{\sqrt{\mu \nu}}\right)+4 \mu \nu-4 \Gamma \sqrt{\mu \nu}=0$

Similarly, for $N_{ \pm}$we have:

$\lambda^{3}+\lambda^{2}(1+\nu-\mu)-\lambda\left(\Gamma \frac{(\nu-\mu)}{\sqrt{\mu \nu}}\right)+4 \mu \nu+4 \Gamma \sqrt{\mu \nu}=0$.

In the following, we describe some of the chaotic behaviors generated by the system of equations (1-3), by exploring the parameter space while $\Gamma$ is kept fixed (in practice $\Gamma=0.9$ ). We consider only situations where the linearization around non-trivial fixed points gives one real eigenvalue, the two others being complex conjugates. The real eigenvalue is always negative, ensuring that the fixed points have a stable direction. The real part of the complex conjugate eigenvalues will depend on the value of $\mu$ and $\nu$.

Among the wide variety of dynamical regimes observed in this simple system, a particularly interesting chaotic behavior can be observed for some parameters (see for instance the chaotic attractor shown in figure 1 obtained for $\mu=0.119, \nu=0.1$ and $\Gamma=0.9)$. For these parameter values, the system explores the phase space around one of the fixed points $N$ for a given time, and chaotically switches from one solution to its opposite, leading to chaotic reversals of amplitudes $D$ and $Q$ ( $V$ does not change sign). Although the evolution during a given polarity is strongly chaotic, reversals exhibit very robust characteristics. In order to understand the mechanisms leading to such dynamics, in particular how the system becomes chaotic, it is useful to keep parameters $\Gamma$ and $\nu$ to these values $(\Gamma=0.9$ and $\nu=0.1$, while only $\mu$ is varied. In addition, the dimension of the problem can be reduced by means of a Poincare map in the phase space [3]. As shown in figure [1. Poincare map is defined as the surface orthogonal to the $(D, Q)$ plane which contains the line joining the two fixed points $N_{+}$and $N_{-}$(this line provides a graduated axis with $O$ the origin). Our first return map is then defined by the intersection $x_{n+1}$ between trajectories of the system (projected in the $(D, Q)$ plane) and this line, as a function of the previous intersection $x_{n}$. Figure 2 shows a bifurcation diagram of $x_{n}$ as $\mu$ is decreased from 0.15 to 0.1 .
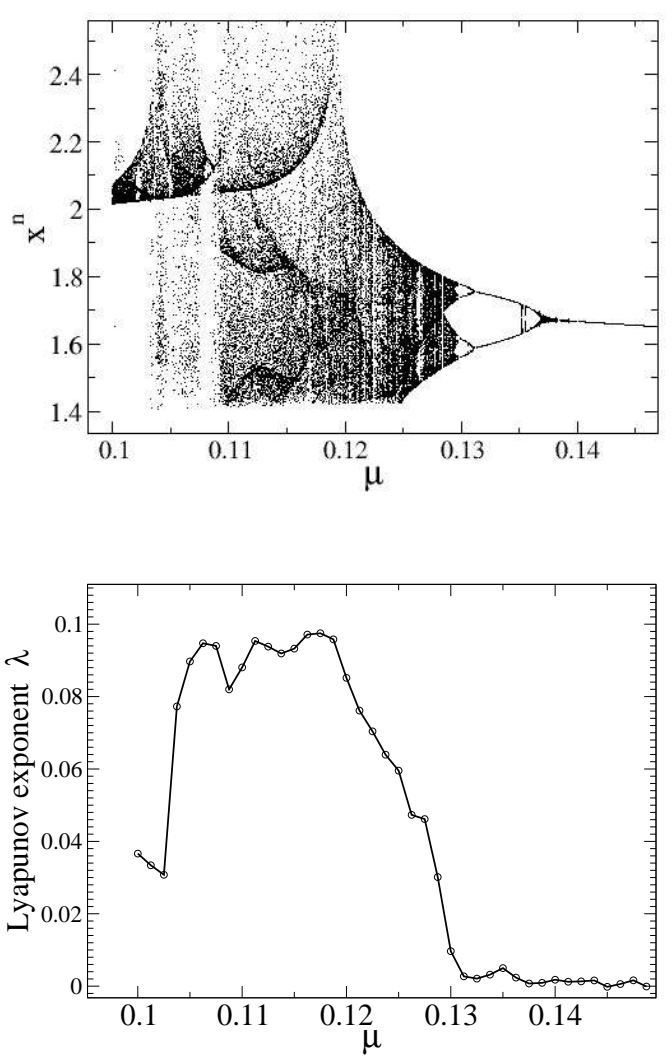

Fig. 2: Top: Bifurcation diagram of the return map $x_{n}$ as a function of $\mu$. When $\mu$ is decreased from 0.15 to 0.1 , the transition to chaos is achieved through a period doubling cascade. Bottom: Evolution of the Lyapunov exponent $\lambda$ of the system when $\mu$ is decreased. The successive period doubling events lead to a chaotic state, characterized by an increase of $\lambda$.

When $\mu>0.2$, two stationary solutions are obtained, corresponding to the fixed points $N_{+}$and $N_{-}$. For $\mu<0.2$, these fixed points loose their stability and a stable periodic orbit is created around each fixed points, corresponding to stable solutions in the iterated map. Below $\mu=0.138$, a series of pitchfork bifurcations is observed in the return map of figure 2 top, due to the successive period doubling bifurcations of the periodic orbit surrounding $N_{+}$. Because of the symmetry of the system, a similar transition occurs around $N_{-}$.

We thus see that our simple dynamical system exhibits a classical route to chaos, by period doubling bifurcations. This scenario is observed in several discrete applications, like the Henon map or the logistic map. Note that the transition to chaos is very sharp, with a Lyapunov exponent $\lambda$ (characterizing the rate of separation of infinitesimally 
$\mu=0.12$
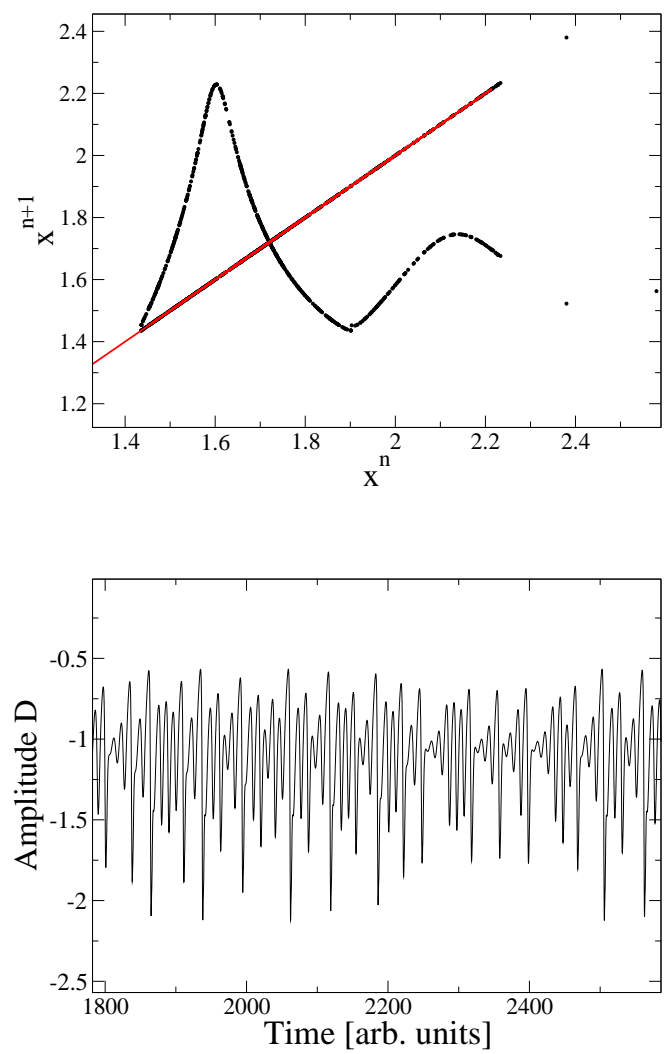

Fig. 3: Numerical integration of the model for $\mu=0.12$, $\nu=0.1$ and $\Gamma=0.9$. These parameters correspond to a fully chaotic state, with trajectories fluctuating around one of the fixed points. Top: discrete map. Bottom: time evolution of $D$. Initial conditions are chosen in the basin of attraction of $N_{+}$.

close trajectories) which rapidly tends to 0.1 at $\mu \approx 0.13$ (see figure 2 bottom).

Figure 3 shows trajectories for $\mu=0.12$, for which a chaotic attractor is obtained. On the iterated map, the system randomly explores the interval $1.4<x_{n}<2.3$ (figure 3. top), defining a typical size of the chaotic attractor, bounded in the phase space $(D, Q, V)$. It is important to note that symmetries of our dynamical system impose that a similar attractor is obtained in the vicinity of the opposite fixed point $N_{-}$. However, for $\mu=0.12$, these two symmetric attractors are disconnected, and there are no reversals (figure 3, bottom). This is a noticeable difference with other deterministic models for reversals. For instance, in the Lorenz model, a period doubling route to chaos can also be observed 11, but in that case, the initial periodic orbit is a cycle surrounding the fixed points of the system, and therefore already involving reversals of the amplitudes. In the next section, we describe how a further decrease of $\mu$ yields chaotic reversals of $D$ and $Q$, very different from reversals obtained in Lorenz or Rikitake models.

\section{Crisis-induced intermittency and reversals}
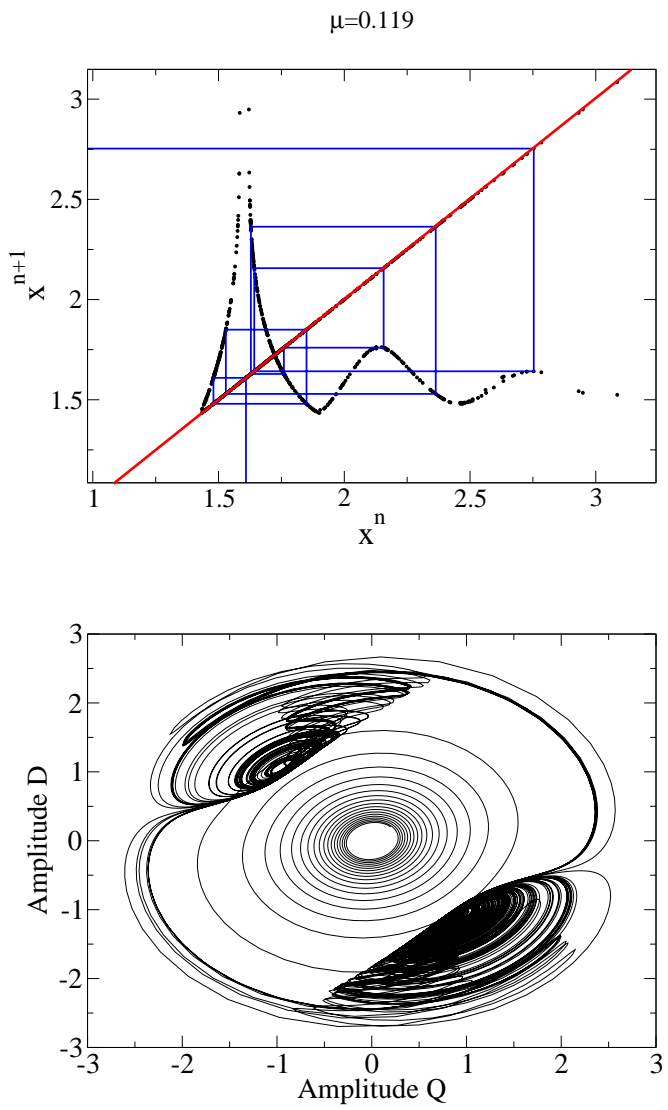

Fig. 4: Dynamics of the model for $\mu=0.119, \nu=0.1$ and $\Gamma=0.9$. Discrete application (top) is very similar to the one obtained for $\mu=0.12$, except for a small region connected to the opposite fixed point. Bottom: evolution of the system in the phase space $(D, Q)$.

For $\mu=0.12$, the system chaotically fluctuates around a fixed point, but without reversing. From this state, a slight decrease of $\mu$ yields a transition to a dynamics of reversals between two symmetric states with well defined values. Figure 4 shows the evolution of the model for $\mu=0.119$, in such a reversing regime. Note that the behavior close to one of the fixed points is similar to the one generated for $\mu=0.2$, i.e without reversals. However, for $\mu=0.119$, the two attractors are now connected, allowing chaotic reversals between the two opposite states.

Changing one of the parameter of the system (here $\mu$ ) yields a discontinuous change of the structure of the attractor. This typical behavior of dissipative systems is known as a crisis phenomenon. The crisis behavior of dynamical systems has been discovered and extensively studied by Grebogi, Ott and York 12, 13, mainly in the context of discrete maps. Following these authors, a crisis is defined as "a collision between a chaotic attractor and a coexisting unstable fixed point or periodic orbit". 
Typically, in a crisis scenario, modifications of the structure of the attractor are of three kinds: in a first case, the chaotic attractor is suddenly destroyed at the critical point. In a second case, the size of the attractor undergoes an abrupt increase. In a last case, two attractors or more collide and a larger attractor is created. In each of these three cases, the structural change is related to a collision with an unstable attractor (chaotic or not), and it is possible to characterize the phenomena with a typical time $\tau$. This $\tau$ measures the finite time during which the system behaves as before the crisis, and then rapidly leaves this region of chaotic transient.

In our dynamical system, the apparition of chaotic reversals seems to belong to the third class, i.e. the merging of two distinct attractors. Let us define $\mu_{c}$ as the critical value of $\mu$ for which reversals appear. When $\mu>\mu_{c}$, the two attractors close to each of the fixed points possess their own basin of attraction, separated by a boundary basin. Depending on the initial conditions, trajectories tend to one attractor or the other, and are trapped in the basin. Because of the symmetry of the system, when $\mu=\mu_{c}$, the two attractors simultaneously collide the boundary basin separating them, which allows a connection between both attractors, through some unknown unstable periodic orbits of the attractors. As a consequence, for $\mu=\mu_{c}$, the system spends a long time exploring chaotically the vicinity of one of the fixed point before it suddenly leaves this region and fluctuates around the opposite solution. The two attractors being symmetrical, the process then repeats and chaotic reversals are generated. We can thus characterize the dynamics by a time $\tau$ which is naturally defined as the average duration between two successive reversals, hereafter referred as Waiting Time Duration (WTD).

In general, Grebogi et al have shown that $\tau$ follows a power law

$$
\tau \sim\left(\mu-\mu_{c}\right)^{-\gamma}
$$

Using numerical simulations, we determined numerically this law. For each value of $\mu, \tau$ is obtained by averaging all the WTDs obtained in a time series containing one thousand reversals.

Figure 5-top shows the averaged waiting time duration $\tau$ between two successive reversals as a function of $\mu$. The curve is diverging for $\mu_{c} \sim 0.11928$. In practice, this critical value has been obtained by noting that no reversals occurred after an integration time $t>1.10^{5}$, whereas reversals were observed for $\mu=0.119275$.

Figure 5 bottom shows how the waiting time durations evolve as the critical point is approached. There is little doubt that this curve follows a power law. However, even if our numerical simulations suggest a critical exponent $\gamma \simeq 1 / 2$, it is difficult to conclude on a precise exponent, since a small error on the determination of $\mu_{c}$ significantly modifies the measured power law.

In some idealized cases, Grebogi et al. [13] have shown that it is possible to obtain the critical exponent $\gamma$ theoretically and to express it as a function of the eigenvalues of the periodic orbits involved in the crisis. In the case of
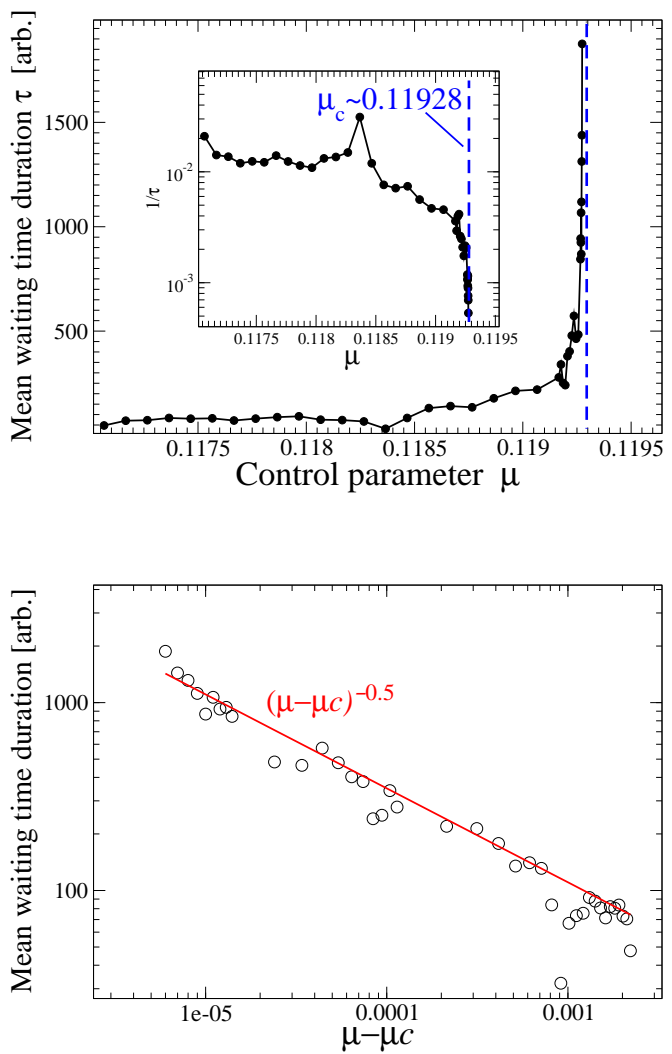

Fig. 5: Evolution of the characteristic waiting time duration between two successive reversals when the critical point of the crisis is approached. Top: $\tau$ as a function of $\mu$, suggesting $\mu_{c}=0.11928$. Inset shows $1 / \tau(\mu)$. Bottom: Evolution in logarithmic scale as a function of $\mu-\mu_{c}$. Numerical simulations suggest an exponent close to $\gamma \sim 1 / 2$.

discrete maps:

$$
\gamma=\frac{1}{2}+\left(\ln \left|\alpha_{1}\right|\right) / \ln \left|\alpha_{2}\right|
$$

where $\alpha_{1}$ et $\alpha_{2}$ are respectively eigenvalues of the unstable and stable manifolds of the two periodic orbits considered. In principle, it should be possible to use this formula to obtain $\gamma$ theoretically from the Poincare map. Unfortunately, the method involves calculating numerically the stable and unstable manifolds of the orbits mediating the crisis, leading to an error bar on the estimate of $\gamma$ too large to be compared to the exponent computed from the waiting time durations. Although the value of $\gamma$ seems very close to the theoretical lower bound, our numerical results are not accurate enough to conclude.

This relation between the exponent and the eigenvalues of the periodic orbits relies on the assumption that the crisis is of the heteroclinic tangent type, which means that it is induced when the unstable manifold of a periodic orbit $(A)$ becomes tangent to the stable manifold of a second unstable periodic orbit $(B)$ (located outside 

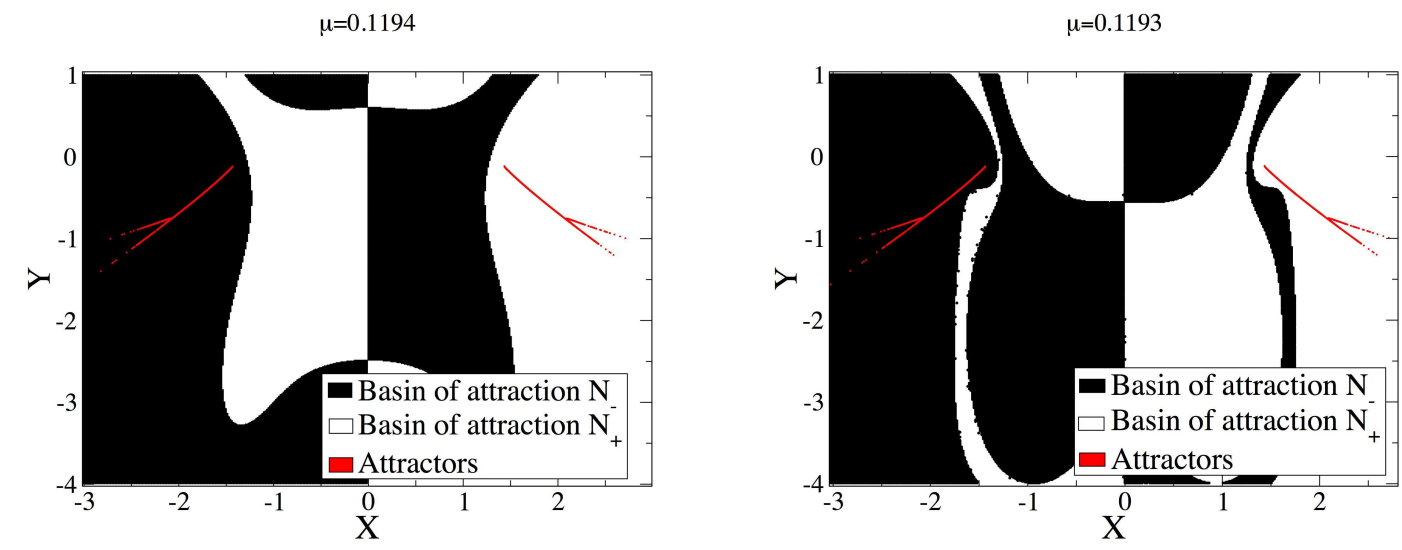

$\mu=0.11928$

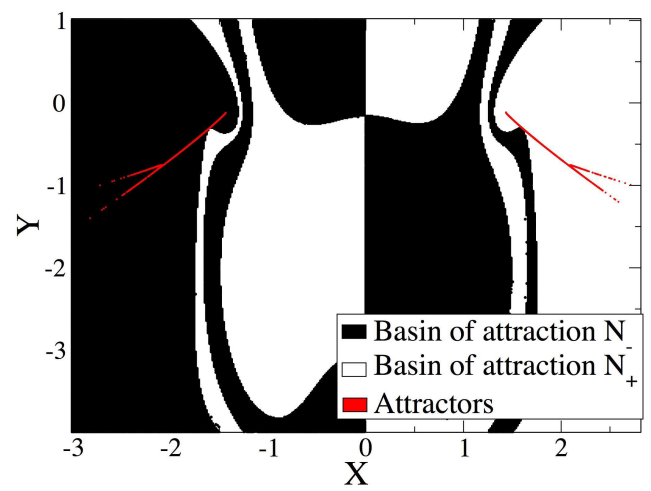

Fig. 6: Basin of attraction of chaotic attractors projected in the Poincare section. White and black colors distinguish the basins of attraction of each attractors. Attractors are shown in red. Top: before the crisis, for $\mu=0.1194$ (left) and $\mu=0.1193$ (right). Bottom: For $\mu=0.11928 \approx \mu_{c}$, we observe a collision between attractors at the basin boundaries, illustrating the boundary crisis.

the basin of attraction before the crisis). One particularity of our dynamical system is that the orbit $(B)$ destroying the first attractor belongs to the basin of attraction of the second attractor. Because of the symmetries, identical connections are achieved with both attractors, allowing the system to chaotically come back to the attractor it previously leaved.

From the Poincare section defined in figure 1, it is possible to visualize the collision leading to the crisis, and figure 6] shows the basin of attraction of the system in the Poincare section $(X, Y)$. Theses basins are numerically obtained by using a grid of $(500 \times 500)$ initial conditions $\left(X_{0}, Y_{0}\right)$ : the point $\left(X_{0}, Y_{0}\right)$ is colored in black (respectively in white) if the computed trajectory starting from this initial condition ultimately tends to the attractor surrounding $N_{-}$(respectively $N_{+}$). Figure 6(a) shows the system before the crisis, for $\mu=0.1194>\mu_{c}$. In red are points corresponding to the intersection of trajectories of the attractor with the Poincare section. Note that the two attractors are distinct. Black and white regions clearly indicate symmetric basins of attraction, and are such that the two attractors are connected only to their own basin. Figure 6(b) corresponds to a slightly smaller value, $\mu=0.1193>\mu_{c}$, showing that a change of $\mu$ toward $\mu_{c}$ yields a change of shape of the basins of attraction and an increase of their size. Finally, for $\mu=0.11928 \approx \mu_{c}$, each of the chaotic attractor collides with the boundary of the basin of attraction of the symmetric attractor. A look at the trajectories in the phase space indicates that the orbit involved in this boundary crisis seems to be an unstable periodic orbit encircling the trivial point $O$. Note indeed that this kind of reversals are only observed when $O$ is an unstable focus. It is interesting to note that when these reversals appear, the largest Lyapunov exponent is not modified, since reversals are due to the fusion of two identical chaotic attractors nearly unchanged by the crisis event. In this regime, we thus obtain a Lyapunov exponent $\lambda \simeq 0.1$.

As shown above, for $\Gamma=0.9$ and $\nu=0.1$, a decrease of $\mu$ leads to four well defined successive behaviors: a stationary state, periodic then chaotic oscillations around this non-trivial fixed point, and finally, chaotic reversals between the vicinity of the two fixed points when $\mu<\mu_{c}$. For further decrease of $\mu$, the averaged waiting time between reversals strongly decreases and the system finally 
reaches a regime of chaotic oscillations. Figure 7 shc the evolution of the system as a function of time and phase space, for $\mu=0.101, \nu=0.1$ and $\Gamma=0.9$. Whes finally reaches the value of $\nu$, we note that the system comes linearly stable at the origin $O$, since the real pe of $\lambda_{1}$ and $\lambda_{2}$ vanish. However, this bifurcation appears be strongly subcritical: for initial conditions chosen ins the basins of attraction of the non-trivial fixed points, trajectories stay inside this basin of attraction. The $s$ tem stabilizes on the trivial fixed point $O$ independer of initial conditions only for $\mu<0.063$.
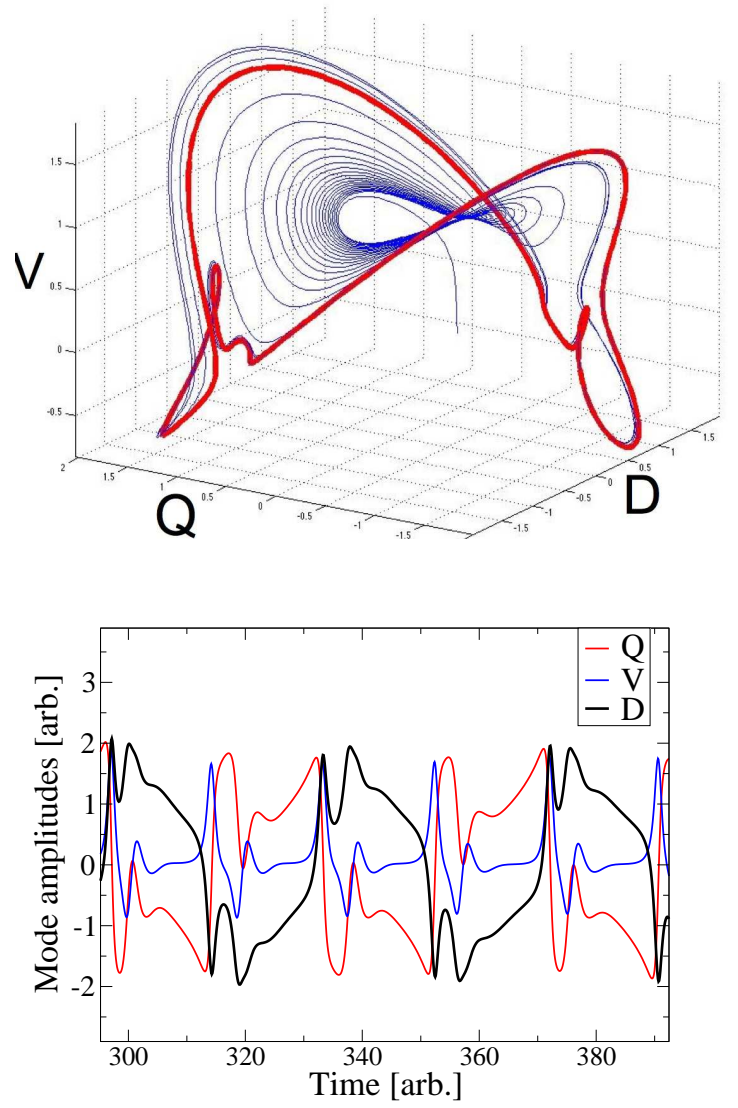

Fig. 7: Top: phase space for $\mu=0.101, \nu=0.1$ and $\Gamma=$ 0.9. Reversals are not chaotic anymore for these values. A periodic connection is achieved between the two fixed points. Bottom: Time evolution of the three amplitudes $D, Q, V$.

Inside this interesting regime of bistability, a series of transitions is observed in the dynamics of our system as $\mu$ is decreased. As it can be seen in figure 8 , for $0.065<$ $\mu<0.1$, the system explores the vicinity of one of the fixed point during a given time and suddenly makes an oscillation around the trivial point $O$. Although it stays on the basin of attraction of the same attractor, the trajectory seems to briefly explore the opposite state. During the time spent close to the fixed point, the behavior can be slightly chaotic. We thus observe an intermittency be-
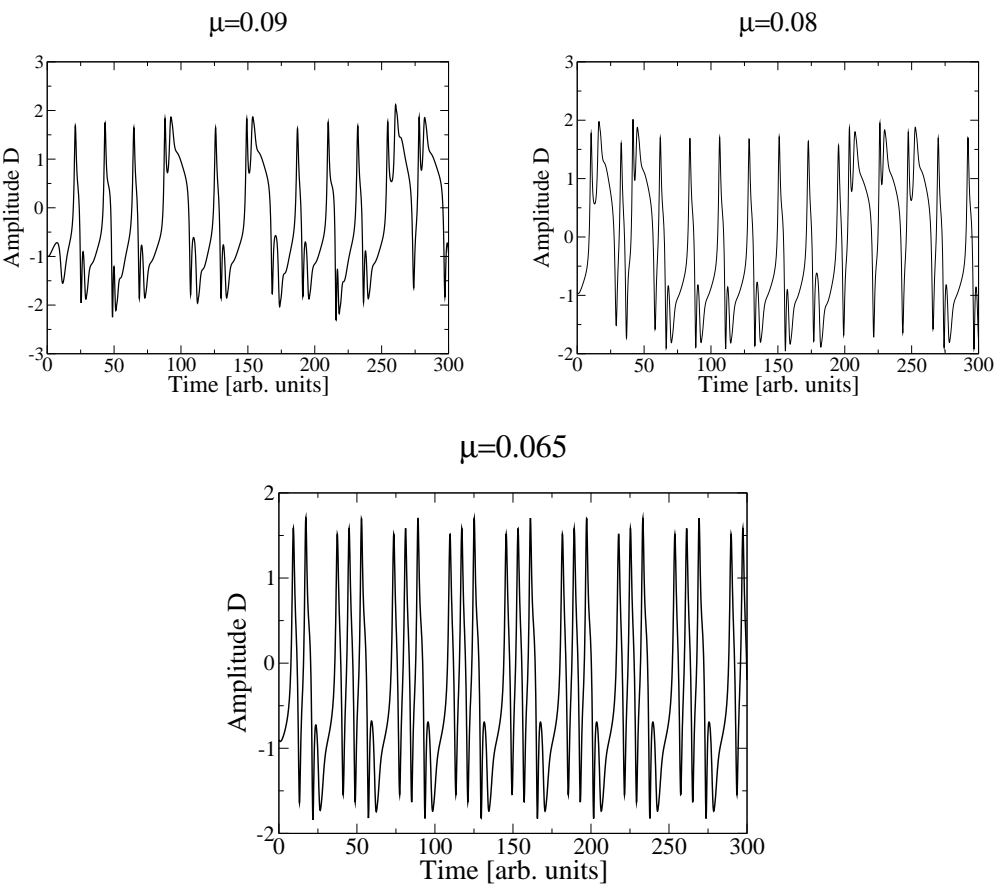

Fig. 8: Evolution of the amplitude $D$ for different values of $\mu$. Top, left: $\mu=0.09$. Top, right: $\mu=0.08$. Bottom: $\mu=0.065$. Note the asymmetric behavior of the dynamics in the latter case. However, the symmetric attractor can be obtained with different initial conditions.

tween a chaotic state around a fixed point and an unstable periodic orbit: as time evolves, the system switches chaotically from the vicinity of the fixed point to the unstable periodic orbit encircling the origin. As expected, the mean time between these switches increase when $\mu$ decreases. For $\mu=0.065$, surprising asymmetric reversals can be observed, despite the symmetry of equations.

The set of parameters chosen so far ensures the existence of only two of the four non-trivial fixed points. We have seen that this is already sufficient to generate several different dynamical regimes, mainly related to reversals. One interest of this model is its ability to describe very different regimes, without any needs of nonlinear terms of higher degrees. This can be illustrated by choosing parameters such that $\sqrt{\mu \nu}-\Gamma>0$, meaning that there are now four non-trivial fixed points in addition to $O$. In this case, the two additional solutions induce new dynamics. Figure 9 shows the evolution of trajectories in the phase space $(D, Q)$ (top) and as a function of time (bottom), for $\mu=\nu=0.35$ and $\Gamma=0.1$.

Finally, others exotic behaviors can be obtained. For instance, figure 10] shows the evolution of the system for $\mu=0.3, \nu=0.2$ and $\Gamma=5$. In this regime, an intermittency between two distinct behaviors is observed. During a first phase, trajectories slowly escape from the unstable periodic orbit surrounding the trivial point $O$. When the amplitude of the oscillation is sufficiently large, it reaches 

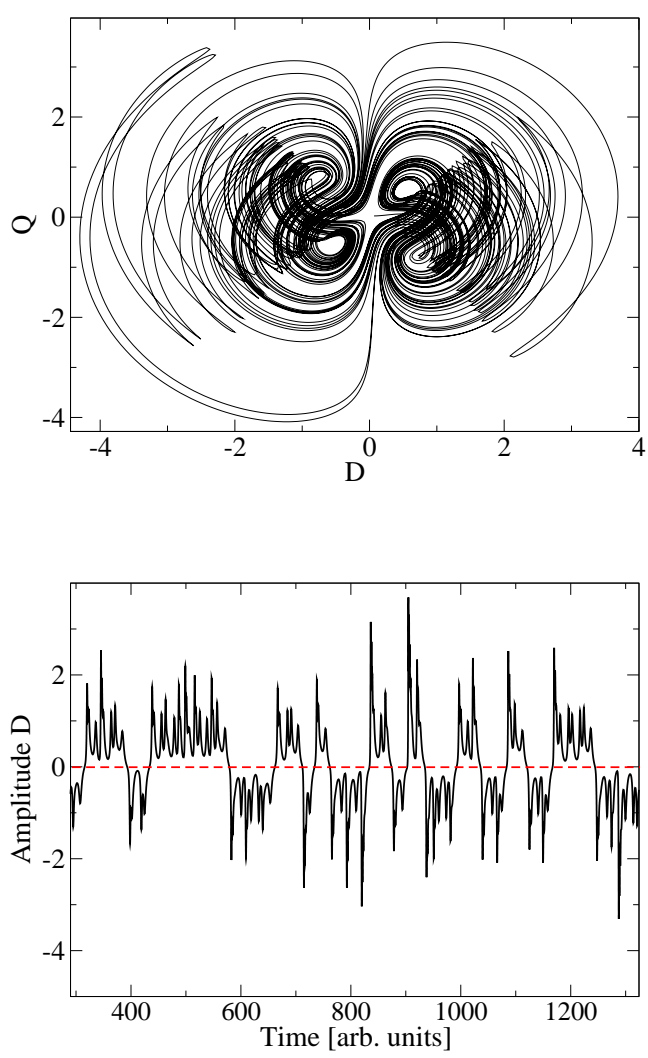

Fig. 9: Reversals obtained for $\mu=\nu=0.35$ and $\Gamma=0.1$, when the four non-trivial fixed points are involved. Top: evolution in the phase space $(D, Q)$. Bottom: evolution of the amplitude $D$ as a function of time.

the basin of attraction of one of the two fixed points, and after some transient wandering around this fixed point, the trajectory is re-injected toward the center of the initial periodic orbit, and the process repeat. Note that here, chaos is essentially due the apparent random choice of the fixed point involved at the end of the oscillations around $O$.

Compared to previous similar models, this simple dynamical system, involving only 3 quadratically coupled equations, generates several different dynamical behaviors, depending on the values of the free parameters $\mu$, $\nu$ and $\Gamma$. It would be interesting to see at which point the different characteristics described here are robust. For instance, one could add multiplicative or additive noise to equations (1-3), or take into account terms of higher degrees to couple the three amplitudes. The present form of the model (without noise and limited to quadratic terms) presents of course the advantage to remain extremely simple, and yet generating complex dynamical regimes.
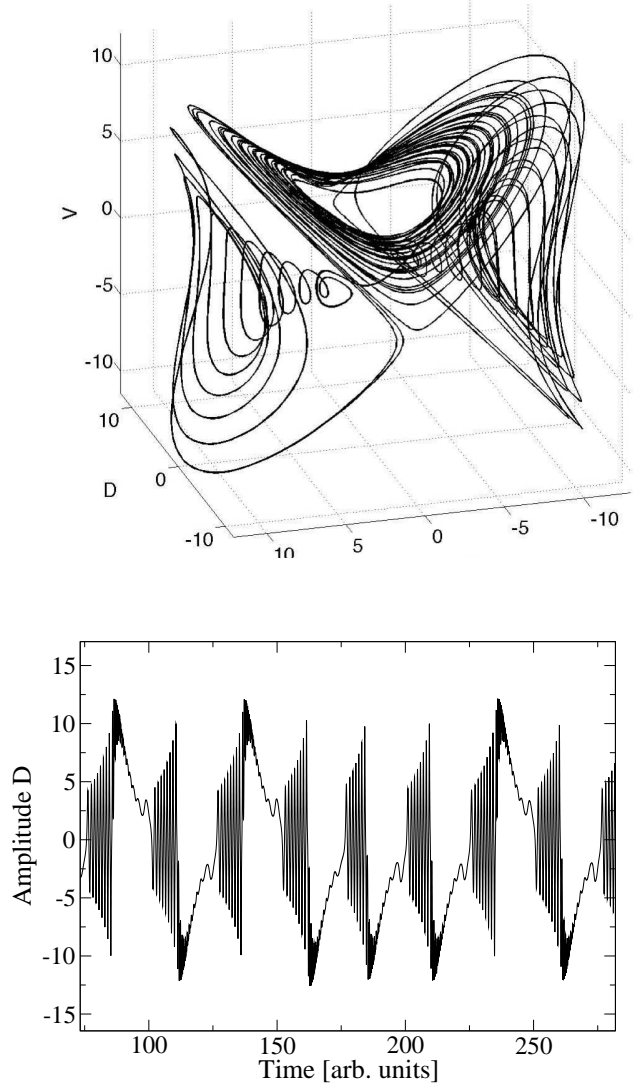

Fig. 10: Evolution of the system for $\mu=0.3, \nu=0.2$ and $\Gamma=5$. Top: evolution in the phase space $(D, Q)$. Bottom: evolution of the amplitude $D$ as a function of time.

\section{Physical interpretation and comparison with geodynamo reversals}

The perspective of a description of the reversals of the Earth's magnetic field in the framework of a low dimensional model is very tempting. Indeed, a low dimensional description provides a simple vision of the phenomena, and allows to make interesting predictions concerning the shape and the statistical properties of the geomagnetic reversals. In fluids dynamos, the small ratio between kinematic viscosity and magnetic resistivity (small magnetic Prandtl number), together with the proximity to the dynamo threshold, strongly reduce the degrees of freedom of the system, and low-dimensional dynamics can be expected. For this reason, this approach has been followed by several studies in the past [9], 6], [14, 15], [16]. Unfortunately, despite their chaotic behaviors, low dimensional deterministic models generally yield too simple dynamics, and fail to realistically reproduce observational data. For instance, Lorenz and Rikitake models exhibit typical growing oscillations between reversals, characteristics of their low-dimensionality, but absent from geomagnetic measurements.

As shown in the previous sections, reversals of our simple model are generated by crisis-induced intermittency, a 

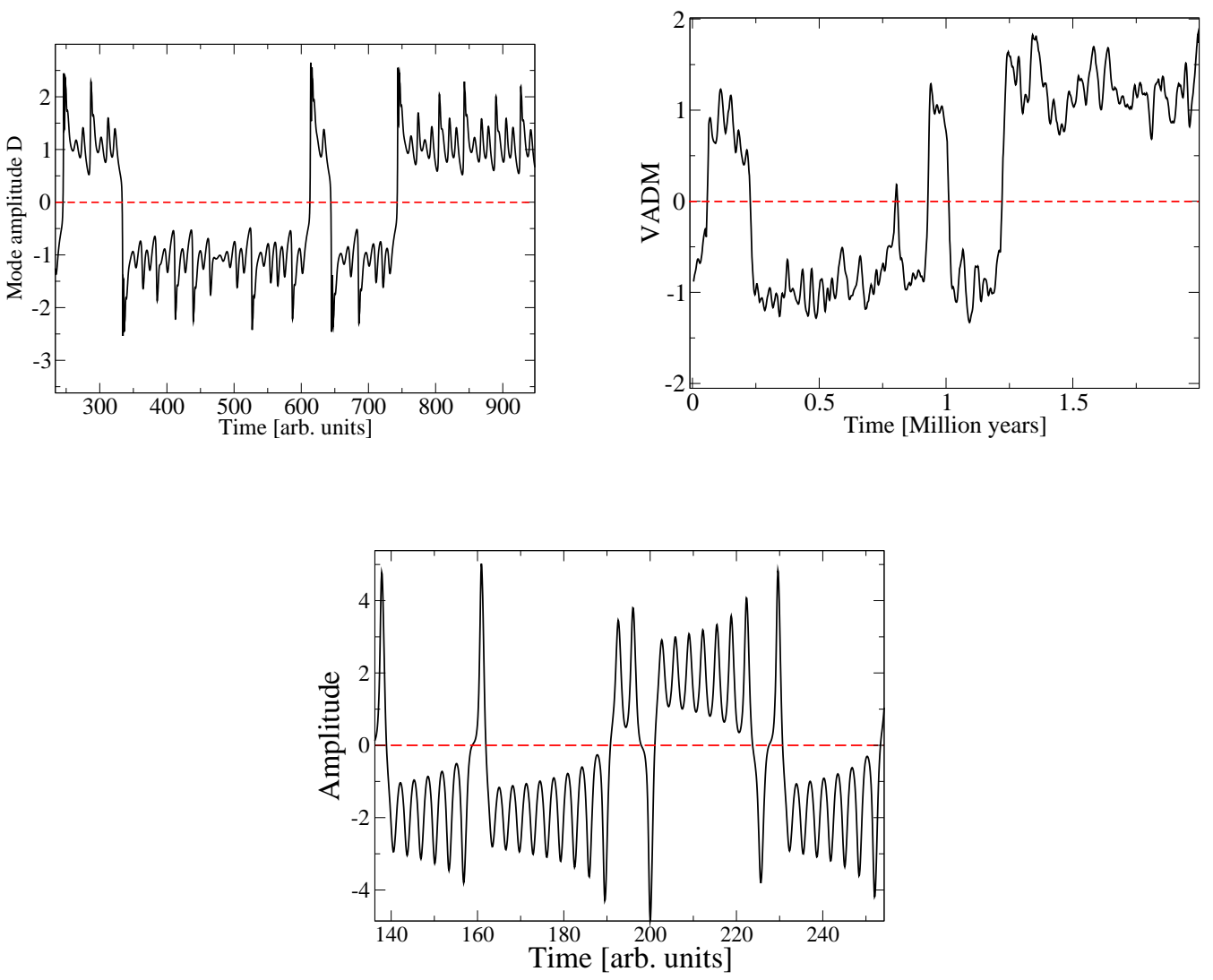

Fig. 11: Comparison between time series obtained from paleomagnetic data (top,right)(SINT2000 data 17]) and from the model $(D, Q, V)$ with $\mu=0.119, \nu=0.1$ and $\Gamma=0.1$ (top,left). A typical time series obtained from the Lorenz model is shown at the bottom.

mechanism different from the one creating chaotic reversals in classical models. We will show in this section that the model (1-3) leads to a better agreement with geodynamo reversals than Lorenz and Rikitake models. Moreover, using symmetry arguments, it is possible to give a simple physical interpretation to the three amplitudes.

The magnetic field of the Earth is mainly dominated by its dipolar component. Figure 11(top-right) shows the evolution of the Earth dipole moment during the past two millions years 17. Although its amplitude is not known during reversals, it has been suggested that the quadrupolar mode could also play an important role during reversals [18, 19]. In the model (1-3), amplitudes $D$ and $Q$ can be regarded as dipolar and quadrupolar components of the magnetic field. Note that the system is invariant with respect to the symmetry $(D \rightarrow-D, Q \rightarrow-Q)$, in agreement with the symmetry $(B \rightarrow-B)$ of the magnetic field $B$ in the equations of magnetohydrodynamics. Another important symmetry in the Earth core is the mirror symmetry with respect to the equatorial plane. Under this symmetry, the dipole is unchanged whereas the quadrupole is transformed in its opposite. This implies that the dipole and quadrupole can not be linearly coupled if the flow in the Earth core is mirror symmetric. By noting that the amplitude $V$ is coupling $D$ and $Q$ in equations (11-3), we can therefore interpret $V$ as a velocity mode (externally forced by $\Gamma \neq 0$ ) which breaks the mirror symmetry of the Earth core flow. The term $Q D$ in equation 3 thus stands for the Lorentz force (quadratic in the magnetic field $B$ ). The system of equations $(1-3)$ therefore models the interaction between dipolar and quadrupolar magnetic modes coupled by a mirror-antisymmetric velocity mode, and can be directly compared to geomagnetic data.

This interpretation of the model is in fact very close to a recent model proposed by Petrelis and Fauve, also relying on a dipole-quadrupole interaction [20], and aiming to model the dynamics observed in the VKS (Von Karman Sodium) experiment, a laboratory turbulent fluid dynamo 21. The VKS experiment exhibits chaotic and periodic reversals of the polarity of the magnetic field, resulting from a surprisingly low-dimensional dynamics 22, and very similar to the predictions of the Petrelis-Fauve model. In this model however, chaos is generated by the addition of a stochastic noise in the equations (modeling the tur- 
bulent fluctuations), and the velocity mode $V$ is not explicitly taken into account. Nevertheless, it is interesting to note that most of the regimes of the VKS experiment can also be reproduced with our simple model, despite its deterministic dynamics 23. This underlines the fact that at small Pm, the characteristics of the turbulent flow and the details of the configuration (such as boundary conditions) can be ignored as far as the dynamical regimes of the magnetic field are considered.

Kono has shown in 1987 that the Rikitake model was unable to reproduce the Probability Density Function (PDF) of waiting times between reversals, or the PDF of the amplitude of the dipolar field 24. In general, distributions of the averaged waiting time between reversals are assumed to be poissonian for the geomagnetic field . It is however difficult to conclude, since only a small number of reversals are available 25. The Rikitake model clearly fails to describe a poissonian process: depending on the parameters, the model predicts either a cutoff forbidding long events, or the apparition of too many long events. We have computed the distribution of the waiting times for $\mu=0.119$, $\nu=0.1$ and $\Gamma=0.9$, and figure 12 shows a comparison to the paleomagnetic data, obtained from the CK95 database 26 . One can see that the very short times are characterized by a quantification. This obviously comes from the minimal time it takes to the system to follow at least one periodic orbit around a given fixed point before reversing. This behavior is typical of models with low-dimensional chaos. For larger waiting times however, a poissonian law is obtained, with a distribution

$$
P(\tau)=\frac{1}{<\tau>} e^{-\frac{\tau}{<\tau>}}
$$

Our model is thus able to reproduce the poissonian behavior observed for the Earth magnetic field, despite the small number of degrees of freedom. Because the quantification at small time is a strong property of deterministic chaos, it would be interesting to search for such a behavior in geomagnetic observations. Note however that this type of behavior is easily destroyed in the presence of noise, which cannot be avoided in the Earth core due to the turbulent fluctuations of the flow.

Similarly, there is a strong disagreement between observations and classical models on the distribution of the intensity of the dipole. The geodynamo exhibits two symmetric maxima around a minimum at zero, but Rikitake and Lorenz models statistically spend too much time close to zero, giving a different distribution. A study of the distribution of the amplitude $D$ in our model during reversals shows a bimodal distribution with a very low probability at the origin, in agreement with geomagnetic observations. This success of the model is mainly due the the type of chaos generated in the model, which produces a time scale separation between the duration of a reversal (related to some unstable periodic orbit) and the waiting time durations (controlled by the distance from the crisis bifurcation point).
From a general point of view, we see that this new model allows to reproduce some of the main characteristics of the geodynamo, like the poissonian process or the distribution of the intensity of the field. As it appears in figure 11. this resemblance between the two systems is also apparent from a simple examination of the time series: during a stable polarity period, both the model and the Earth magnetic field show some chaotic oscillations, with sometimes bigger events called excursions, during which the dipole seems to reverse but finally comes back to its initial value. Moreover, this model reproduces the tendency of the Earth dipolar field to systematically overshoot its quasi-stationary value just after each reversal, and also the time scale separation between the reversals and the stable polarity states. This model also makes some interesting predictions concerning the geodynamo. For instance, when the dipole $D$ goes to zero during a reversal, this corresponds to a strong increase of the quadrupolar component $Q$ in the model (see figure 14). Note that because of the deterministic nature of the model, the reversals always exhibit these features. This predicts that during a reversal, the magnetic field of the Earth would change shape (by transferring the magnetic energy from the dipolar to the quadrupolar component), rather than simply vanishing.

In addition, this model relies on the existence of a velocity mode $V$ breaking the mirror symmetry of the Earth core flow. Several studies seem to confirm this role of an equatorially antisymmetric velocity mode in the geodynamo reversals. First, it has recently been observed that the ends of superchrons (large period of time without geomagnetic reversals) correspond to major flood basalt eruptions due to large thermal plumes ascending through the mantle 27. In the framework of our model, these plumes correspond to an increase in the symmetry breaking of the flow ( $\Gamma$ is increased). In agreement with this observation, it has been shown in global numerical simulations that taking an heterogeneous heat flux (rather than homogeneous) at the core-mantle boundary of the Earth strongly influences the frequency of magnetic field reversals 28. Again, this corresponds in the model to change the forcing of the antisymmetric velocity mode $V$.

\section{Conclusion}

In this paper, we have presented a new deterministic model for reversals. In this model, chaotic reversals are generated through a boundary crisis between two symmetric chaotic attractors. Compared to previous deterministic models, this mechanism produces a relatively complex behavior in a given polarity, while reversals exhibit a very robust shape. In addition, we have shown that several very different dynamical regimes can be obtained, depending on the values of the free parameters of the system.

This model has been compared to reversals of the Earth magnetic field, and a good agreement has been obtained. Although geomagnetic reversals are certainly more complicated than a simple interaction between three modes, 

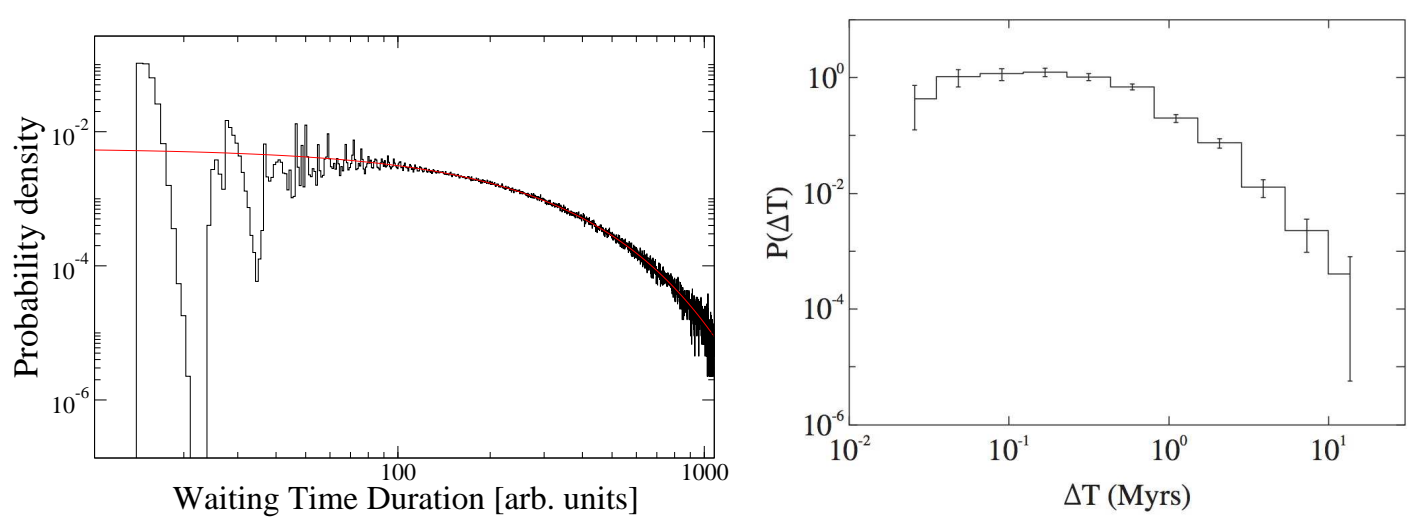

Fig. 12: Left: Distribution of the waiting time durations (WTD) for the model. We observe large oscillations at small time corresponding to the quantification of the unstable periodic orbits, and a poissonian behavior at large time. The red curve shows an exponential fit. Right: Probability distribution of waiting times between reversals for the geomagnetic field, showing an approximative poissonian law (from CK95 database [26]).
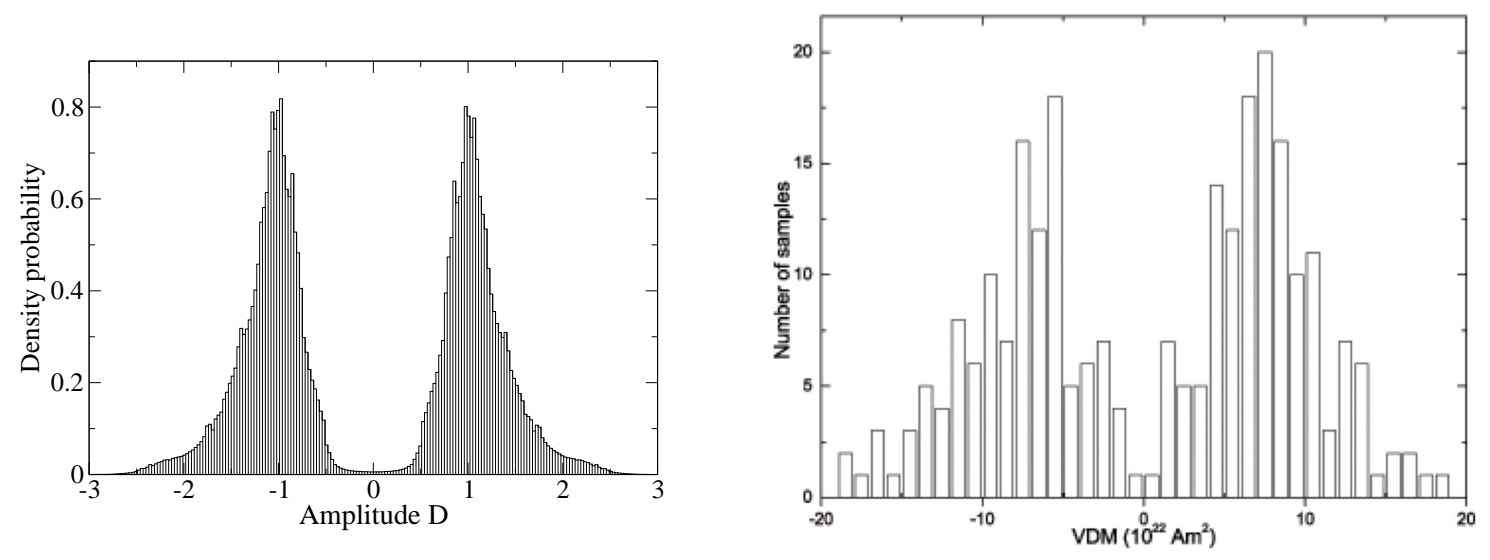

Fig. 13: Left: distribution of probability of amplitudes $D$. Right: bimodal probability distribution of the intensity of the Earth dipolar field.

it is interesting to obtain a simple way to describe this complex phenomenon. The strong similarities between our model and paleomagnetic observations suggest an interpretation of geomagnetic reversals as resulting from an interaction between the dipolar and the quadrupolar components of the magnetic field. In addition, our model predicts that a velocity mode could play the role of the amplitude $V$, by coupling the two magnetic components dipole and quadrupole. It would be interesting to study the different possibilities for such a flow, corresponding to a breaking of the mirror symmetry of the large scale flow in the Earth core. Because of the fast rotation of the Earth, the geostrophic equilibrium makes difficult a spontaneous breaking of this symmetry. However, the presence of the core, decoupling the north and the south hemispheres inside the tangent cylinder, makes possible the existence of large scale antisymmetric velocity modes: for instance, an heterogeneous heat flux at the core-mantle boundary may force the large scale velocity mode $V$.
Several physical systems, different from the dynamo, display chaotic reversals of an observable between two symmetric states. In general, the interpretation in terms of a few coupled equations generating deterministic chaos is easily ruled out. Indeed, most of the models predict a behavior far too simple between reversals, like the growing periodic oscillations of the Lorenz or Rikitake equations. It is possible to attenuate this behavior by adding noise to the system, taking into account higher order terms, or increasing the dimension of the model. The new model presented in this article shows that it is in fact possible to obtain a more complex behavior between reversals, closer to stochastic models predictions, with only three coupled equations limited to quadratic orders. This is an important observation, because it opens the perspective to understand the dynamics of complex physical systems using very simple mechanisms, mainly relying on symmetry arguments. 


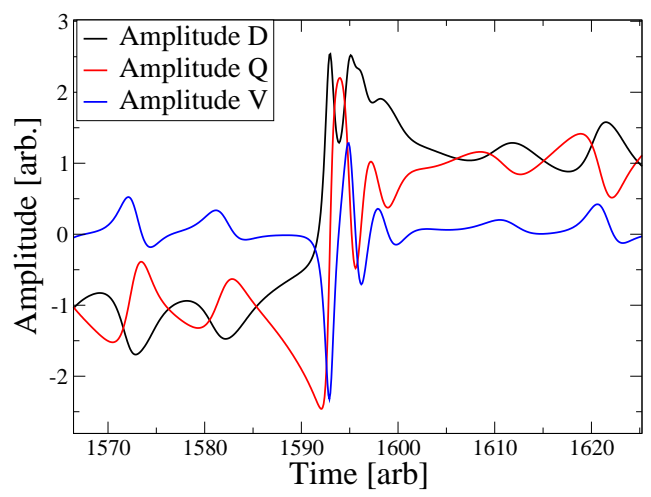

Fig. 14: Evolution of the three components of the model during a reversal, for $\mu=0.119, \nu=0.1$ and $\Gamma=0.9$. Note the rapid time scale related to the reversal event, compared to the rest of the dynamics. When the dipole $\mathrm{D}$ goes to zero during the reversal, the magnetic energy is transferred to the quadrupolar component of the magnetic field. After the reversal, the dipole systematically overshoots its mean value.

The author would like to sincerely thank Stephan Fauve, Francois Petrelis and Emmanuel Dormy for their very inspiring discussion and constructive comments. This work was supported by the NSF under grant AST-0607472 and by the NSF Center for Magnetic Self-Organization under grant PHY-0821899.

\section{References}

1. S. Wiggins, Introduction to applied nonlinear dynamical systems and Chaos, Springer, Berlin (1990)

2. E. отт, Chaos in dynamical systems, Cambridge Univ. Press, Cambridge U.K. (1993)

3. J. Guckenheimer And P. Holmes, Nonlinear oscillations, dynamical systems, and bifurcations of vector fields, Applied Mathematical Sciences, New York: Springer (1982)

4. E.N. Lorenz, Deterministic non-periodic flow, J. Atmos. Sci. 20, 130-141 (1963)

5. O.E. Rossler, Phys. Rev. Lett. 57A, 397 (1976)

6. T. Rikitake, Math. Proc. Cambridge Philos. Soc., 54 89105 (1958)

7. J.C. Sprott, Phys. Rev. E, 50, 647 (1994)

8. C. Gissinger, E. Dormy, S. Fauve, Euro. Phys. Lett. 90 49001 (2010)

9. P. Nozières, Phys. Earth Planet. Inter., 17, 55 (1978)

10. D. Hughes And M.R.E. Proctor, Nonlinearity, 3 (1990) 127.

11. P. Smith, Explaining chaos, Cambridge University Press, Cambridge UK(1998)

12. C Grebogi, E Ott, J.A. Yorke, Phys. Rev. Lett. 48, 1507-1510,(1982)

13. C Grebogi, E Ott, F Romeira, J.A. Yorke, Phys. Rev. A,36, 5365-5380 (1987)
14. I. Melbourne, M.R.E. Proctor And A.M. RuckLIDGE, Dynamo and Dynamics, A Mathematical Challenge, edited by P. Chossat et al. (Kluwer Academic Publishers) 2001, p. 363

15. P. Hoyng, M. A. J. H. Ossendrijver, D. Schmidt, Geophys. Astroph. Fluid Dyn. 94 (2001) 263-314.

16. F. Petrelis, S. Fauve, E. Dormy, J.P. Valet, Phys. Rev. Lett., 102 (2009) 144503.

17. J.P. Valet, L. Meynadier and Y. Guyodo Y, Nature, 435, $802(2005)$

18. P. L. McFadden et AL., J. Geophys. Res. 96, 3923 (1991)

19. G. Glatzmaier and P. Roberts, Phys. Earth Plan. int., 91, 63-75 (1995)

20. F. Petrelis and S. Fauve, J. Phys. Condens. Matter 20, 494203 (2008).

21. R. Monchaux et AL., Phys. Rev. Lett. 98, 044502 (2007);

22. M. Berhanu Et AL., Europhys. Lett. 77, 59001 (2007).

23. C. Gissinger, PhD Thesis, Pierre and Marie Curie University (2010)

24. M. Kono, Geoph. Res. Lett.14, 21-24 (1987)

25. V. Carbone et AL., Phys. Rev. Lett. 96, 128501 (2006)

26. S.C. Cande, and D.V. Kent, J. Geophys. Res. 100, 6093 (1995)

27. V. Courtillot and P. Olson, Earth Planet. Sci. Lett. 260, 495 (2007).

28. G. Glatzmaier et al., Nature (London) 401, 885 (1999) 